\title{
Transitioning from parenteral treprostinil to inhaled treprostinil in patients with pulmonary arterial hypertension
}

\author{
Amresh Raina, James C. Coons, Manreet Kanwar, Srinivas Murali, George Sokos, and Raymond L. Benza \\ Allegheny General Hospital, West Penn Allegheny Health System, Pittsburgh, Pennsylvania, USA
}

\begin{abstract}
Treprostinil is a potent prostacyclin vasodilator indicated for the treatment of pulmonary arterial hypertension (PAH, World Health Organization Group I). Previously, treprostinil was available only in subcutaneous (SC) or intravenous (IV) formulations. Availability of an inhaled formulation of treprostinil has provided clinicians with an alternative to continuous SC or IV treprostinil in appropriate patients. Stable PAH patients whose quality of life has been dramatically impacted by side effects of parenteral therapy or those who have had recurrent, life-threatening bloodstream infections but are otherwise responding well to treatment may be the candidates for continuing prostacyclin therapy with inhaled treprostinil. However, there is little clinical experience with transitioning patients from parenteral to inhaled treprostinil. We present the results of two cases that highlight important considerations in transitioning patients from parenteral to inhaled therapy, including the pharmacologic and clinical equivalence of formulations, dose titration of formulations and suggested criteria for patient selection.
\end{abstract}

Key Words: case study, prostacyclin, prostanoid, pulmonary arterial hypertension, transition, treprostinil

Pulmonary arterial hypertension (PAH) is a chronic, progressive disorder of the pulmonary microvasculature characterized by vasoconstriction, proliferation of pulmonary vascular cells and in situ thrombosis, ultimately leading to right heart failure and death ${ }^{[1]}$ Prostanoids have remained the gold standard for the treatment of PAH since the introduction of intravenous (IV) epoprostenol and clinicians currently have additional prostanoids and routes of administration at their disposal. ${ }^{[2]}$

Treprostinil (Remodulin; United Therapeutics Corporation, Research Triangle Park, N.C.) is a prostacyclin analogue that was approved for subcutaneous (SC) administration by the US Food and Drug Administration in 2002 and IV administration in 2004. In clinical studies, SC and IV treprostinil have demonstrated improvements in exercise capacity, functional class, hemodynamics and survival (compared with historic National Institutes of Health registry data). ${ }^{[3-6]}$ Treprostinil has demonstrated efficacy in the treatment of PAH when administered by either parenteral route. However, infusion site pain, a common side effect associated with SC treprostinil ${ }^{[7]}$ and risk of bloodstream infection with IV treprostinil ${ }^{[8]}$ have limited the appeal of parenteral treprostinil for some patients.

In 2009, an inhaled formulation of treprostinil was approved for the treatment of PAH. In a randomized clinical trial of inhaled treprostinil (Tyvaso, United Therapeutics Corporation), 12 weeks of inhaled treprostinil administered four times daily (q.i.d.) to patients on background bosentan or sildenafil improved 6-Minute Walk Distance (6 MWD), quality of life and $\mathrm{N}$-terminal pro-B-type natriuretic peptide (NT-proBNP) levels. ${ }^{[9]}$ Although bioequivalence between the SC and IV routes has been established for treprostinil, ${ }^{[10]}$ it is unclear whether the inhaled formulation given q.i.d., with associated peaks and troughs of treprostinil concentrations in the blood, will be adequate to maintain patients at an equivalent level of disease status as compared

\begin{tabular}{|l|l|}
\hline \multicolumn{3}{|c|}{ Access this article online } \\
\hline Quick Response Code: & Website: www.pulmonarycirculation.org \\
\hline & DOI: 10.4103/2045-8932.109926 \\
\cline { 2 - 3 } & $\begin{array}{l}\text { How to cite this article: Raina A, Coons JC, } \\
\text { Kanwar M, Murali S, Sokos G, Benza RL. } \\
\text { Transitioning from parenteral treprostinil to } \\
\text { inhaled treprostinil in patients with pulmonary } \\
\text { arterial hypertension. Pulm Circ 2013;3:116-20. }\end{array}$ \\
\hline
\end{tabular}


with parenteral therapy. Furthermore, it is not clear whether it is serum concentrations or pulmonary tissue concentrations of treprostinil that are more important for clinical efficacy. As treprostinil administered by inhalation is delivered directly to the site of disease, it may be that lower serum concentrations of treprostinil are sufficient to sustain treatment efficacy. Notably, there is little clinical experience transitioning patients from parenteral treprostinil to inhaled treprostinil. We report our experience transitioning from parenteral to inhaled treprostinil in two carefully selected PAH patients.

\section{CASE REPORTS}

Patient 1 was a 69-year-old Caucasian woman with a 6-year history of idiopathic PAH. She was initiated on PAH therapy with bosentan $125 \mathrm{mg}$ twice daily (b.i.d.) in October 2005 and sildenafil $20 \mathrm{mg}$ three times daily (t.i.d.) in January 2007. She remained symptomatic, however, with worsening hemodynamics. As a result, IV treprostinil was initiated in April 2008, which was associated with significant improvement in her symptoms and hemodynamics. Unfortunately, she developed recurrent life-threatening central line infections while on IV treprostinil in 2009 and was ultimately transitioned to SC treprostinil (maximum dose $38 \mathrm{ng} / \mathrm{kg} / \mathrm{min}$ ) in January 2010. She experienced intractable infusion site pain on SC treprostinil despite topical anesthesia and analgesics, which markedly impacted her quality of life. After extensive discussion, rather than discontinue prostacyclin therapy, she was electively admitted for transition from SC to inhaled treprostinil in April 2010. A right-heart catheterization was performed pre-and post-transition to assess changes in hemodynamics. Inhaled treprostinil was initiated at three breaths every six hours and increased by three breaths daily to a maximum of nine breaths every 6 hours over three days. Simultaneously, the SC treprostinil infusion dose was decreased by $5 \mathrm{ng} / \mathrm{kg} / \mathrm{min}$ every six hours until cessation of SC treprostinil, with minimal side effects. The total time for SC to inhaled transition was approximately 72 hours. Clinical parameters before and after the transition to inhaled treprostinil are noted in Table 1.

Patient 2 was a 46-year-old Caucasian man with an 8-year history of PAH secondary to $\beta$-thalassemia and cirrhosis with splenectomy. He was initiated on PAH therapy with SC treprostinil and up-titrated to $61 \mathrm{ng} / \mathrm{kg} / \mathrm{min}$ in April 2003. Sildenafil $20 \mathrm{mg}$ t.i.d. was added in July 2006 and bosentan $125 \mathrm{mg}$ b.i.d was added in January 2008. His hemodynamics remained stable on this regimen. However, he developed progressive infusion site pain and serial SC abscesses with life-threatening bacteremia in late 2009 and early 2010. He was offered conversion to IV
Table 1: Hemodynamic and clinical characteristics before and after initiation of inhaled treprostinil (patient 1)

\begin{tabular}{lcc} 
& $\begin{array}{c}\text { Before initiation } \\
\text { of inhaled } \\
\text { treprostinil }\end{array}$ & $\begin{array}{c}\text { 5 months after } \\
\text { initiation of } \\
\text { inhaled treprostinil }\end{array}$ \\
\hline Mean RAP, mm Hg & 1 & 2 \\
Mean PAP, mm Hg & 22 & 17 \\
Mean PCWP, mm Hg & 1 & 8 \\
PVR, Wood unit & 2.7 & 1.6 \\
CI (Fick), L/min/m 2 & 4.2 & 3.2 \\
NT-proBNP, pg/mL & 141 & 105 \\
6 MWD, m & 439 & 502 \\
WHO FC & II & II \\
Reveal score & 4 & 3 \\
\hline
\end{tabular}

CI: cardiac index; FC: functional class; 6MWD: 6-minute walk distance; NT-proBNP: N-terminal pro-B-type natriuretic peptide; PAP: pulmonary arterial pressure; PCWP: pulmonary capillary wedge pressure; PVR: pulmonary vascular resistance; RAP: right atrial pressure; $\mathbf{S v O}_{2}$ : mixed venous oxygen saturation; WHO: World Health Organization; REVEAL: registry to evaluate early and long-term pulmonary arterial hypertension disease management

prostanoid therapy, but he refused given concern over continued infectious risk. Again, rather than discontinuing prostacyclin therapy, the patient was offered transition to inhaled treprostinil in the knowledge that this might not be feasible given his relatively high dose of SC treprostinil. He was admitted in April 2010 for transition from SC to inhaled treprostinil with continuous hemodynamic monitoring in light of the higher dose of SC treprostinil. Inhaled treprostinil was initiated at three breaths every six hours and increased by three breaths daily to a maximum of nine breaths every $6 \mathrm{~h}$ over a period of three days. Simultaneously, the SC treprostinil infusion dose was decreased by $5 \mathrm{ng} / \mathrm{kg} /$ min every six hours until cessation of SC treprostinil by Day 4, with minimal side effects. Clinical parameters, measured before and after the transition to inhaled treprostinil, are noted in Table 2.

\section{DISCUSSION}

Of the three drug classes represented in the treatment of $\mathrm{PAH}$, the prostacyclin class is the best characterized, with therapy-induced improvements in patient functional capacity $^{[3-6]}$ and survival. ${ }^{[11]}$ However, IV prostanoids require placement of a central venous catheter, placing the patient at a risk of rare, but potentially fatal, line infections. Although SC treprostinil does not require an indwelling catheter, a majority of patients experience significant pain at the infusion site, ${ }^{[7]}$ as noted in the preceding cases, thereby limiting its use. Inhaled treprostinil presents a potential option in patients who are unable to tolerate the parenteral forms of treprostinil, but in whom continuation of prostanoid therapy is medically indicated.

Recently, Perez et al. reported their experience of transitioning 18 patients with WHO Group I PAH from parenteral prostacyclins to inhaled treprostinil at six 
Table 2: Hemodynamic and clinical characteristics before and after initiation of inhaled treprostinil (patient 2)

\begin{tabular}{lcccc} 
& $\begin{array}{c}\text { Before initiation of } \\
\text { inhaled treprostinil }\end{array}$ & $\begin{array}{c}\text { Day 1 after initiation } \\
\text { of inhaled treprostinil }\end{array}$ & $\begin{array}{c}\mathbf{2} \text { months after initiation } \\
\text { of inhaled treprostinil }\end{array}$ & $\begin{array}{c}\mathbf{5} \text { months after initiation } \\
\text { of inhaled treprostinil }\end{array}$ \\
\hline Mean RAP, mm Hg & 13 & 5 & 8 & 1 \\
Mean PAP, mm Hg & 52 & 46 & 42 & 35 \\
Mean PCWP, mm Hg & 11 & 8 & 6 & 4 \\
PVR, Wood units & 7.7 & 8 & 6.3 & 5.3 \\
CI (Fick), L/min/m & 2.8 & 2.4 & 3.1 & 3.1 \\
NT-proBNP, pg/mL & 806 & NA & NA & 177 \\
6 MWD, m & 440 & NA & 482 & NA \\
WHO FC & II & II & 7 & II \\
Reveal score & 7 & & 7 & \\
\hline
\end{tabular}

CI: cardiac index; FC: functional class; 6MWD: 6-minute walk distance; NA: not available; NT-proBNP: N-terminal pro-B-type natriuretic peptide;

PAP: pulmonary arterial pressure; PCWP: pulmonary capillary wedge pressure; PVR: pulmonary vascular resistance; RAP: right atrial pressure; SvO $\mathbf{2}_{2}$ : mixed venous

oxygen saturation; WHO: World Health Organization; REVEAL: registry to evaluate early and long-term pulmonary arterial hypertension disease management

tertiary PAH referral centers. ${ }^{[12]}$ Patients were mainly WHO Functional Class II or III and had been stable on prostacyclin therapy for a mean of 110 months prior to transition. The mean dose of IV/SC treprostinil was $73 \mathrm{ng} / \mathrm{kg} / \mathrm{min}$ and epoprostenol was $10 \mathrm{ng} / \mathrm{kg} / \mathrm{min}$. However, at seven months of clinical follow-up, $21 \%$ of patients had worsening PAH and clinical symptoms requiring reinitiation of parenteral therapy, indicating that transition should be approached with caution even at experienced centers.

Insights into the challenges associated with transitions from parenteral to inhaled treprostinil are based upon pharmacokinetic (PK) differences between continuous and intermittent delivery of treprostinil. Treprostinil exhibits a terminal elimination half-life of approximately four hours $\mathrm{h}^{[13]}$ and is associated with peak and trough concentrations when administered q.i.d. by inhalation. Clinical differences between peak and trough 6-MWD in the TRIUMPH study provide rationale for suspecting variable concentrations of treprostinil in the blood and the pulmonary tissue and may have clinical implications for patients considering transitioning from continuous to intermittent therapy. ${ }^{[9]} \mathrm{In}$ that study, the 6 MWD at 12 weeks was improved by $20 \mathrm{~m}$ with peak exposure (measured 10-60 min post dosing) compared with $14 \mathrm{~m}$ at trough exposure (measured $\geq 4 \mathrm{~h}$ post dosing). ${ }^{[9,12]}$

Insight into appropriate transition from parenteral to inhaled treprostinil may be extrapolated from a randomized, single-blind, dose-escalation study evaluating the PK profile of inhaled treprostinil. ${ }^{[14]}$ Sixteen of the first 31 patients in this trial were randomized to receive doses of either $30 \mu \mathrm{g}$ of inhaled treprostinil or placebo, whereas subsequent patients received inhaled treprostinil doses of $60 \mu \mathrm{g}(n=6)$, $90 \mu \mathrm{g}(n=6)$ and $120 \mu \mathrm{g}(n=3)$. Plasma concentrations and hemodynamic parameters were monitored over $180 \mathrm{~min}$. The mean maximum concentration (Cmax) post inhalation was seen within 10-15 minutes and was proportional to doses up to $90 \mu \mathrm{g}$. The mean Cmax values for the 60- and $90-\mu \mathrm{g}$ doses (1.59 and $1.74 \mathrm{ng} / \mathrm{mL}$, respectively) were consistent with those for SC and IV treprostinil at doses of $15 \mathrm{ng} / \mathrm{kg} / \mathrm{min}$ given for $150 \mathrm{~min}$ in healthy volunteers (1.47 and $1.57 \mathrm{ng} / \mathrm{mL}$, respectively). ${ }^{[15]} \mathrm{In}$ addition, treprostinil given by SC infusion at $15 \mathrm{ng} / \mathrm{kg} /$ min over 28 days in healthy volunteers led to a mean Cmax of $1.56 \mathrm{ng} / \mathrm{mL}^{[16]}$ The dose proportionality of SC and IV treprostinil has been previously demonstrated to be linear at therapeutic doses up to $125 \mathrm{ng} / \mathrm{kg} / \mathrm{min} \cdot{ }^{[17]}$ This relationship is also observed with inhaled treprostinil up to a $90-\mu \mathrm{g}$ dose. ${ }^{[12]} \mathrm{A}$ dose of $120 \mu \mathrm{g}$ resulted in significant oxygen desaturation, which may be consistent with an augmented Cmax compared with the lower doses. ${ }^{[14]}$ The target dose of inhaled treprostinil approved by the Food and Drug Administration, however, is $54 \mu \mathrm{g}$ (nine breaths; $6 \mu \mathrm{g}$ per breath) ${ }^{[12]}$ At the target maintenance dose of $54 \mu \mathrm{g}$, the mean Cmax was reported in two studies to be $0.91-1.32 \mathrm{ng} / \mathrm{mL}$.

In the current series, we have described a relatively simple but rational approach, based on PK data, in which the dose of parenteral treprostinil was decreased in increments of $5 \mathrm{ng} / \mathrm{kg} / \mathrm{min}$ every six hours with simultaneous rapid up-titration of inhaled treprostinil. Inhaled treprostinil was initiated at a dose of three breaths $(18 \mu \mathrm{g})$ q.i.d. on Day 1 and up-titrated to six breaths (36 $\mu$ g) q.i.d. on Day 2 and nine breaths (54 $\mu$ g) q.i.d. on Day 3. Our up-titration protocol called for a more rapid up-titration than that seen in TRIUMPH, in which the target dose was achieved typically in the second week. ${ }^{[9]}$ We were wary, but we did not observe any significant prostacyclin-related (headache, nausea, flushing, diarrhea, dizziness) or administration-related (particularly coughing) side effectsdue to the rapid up-titration of inhaled treprostinil.

SC treprostinil was being delivered at doses of 38 and $61 \mathrm{ng} / \mathrm{kg} / \mathrm{min}$ in Patients 1 and 2 , respectively. Down-titration of SC treprostinil and up-titration of inhaled treprostinil began concurrently with a reduction in the SC dose of $5 \mathrm{ng} /$ $\mathrm{kg} / \mathrm{min}$ every six hours until its discontinuation. Since parenteral administration of treprostinilat $15 \mathrm{ng} / \mathrm{kg} / \mathrm{min}$ would provide a concentration profile likely to exceed the target dose (nine breaths) of inhaled therapy, our transition was based upon this reduction each day in the 
SC dose. For practical purposes, however, we elected to conduct a daily down-titration of $20 \mathrm{ng} / \mathrm{kg} / \mathrm{min}(5 \mathrm{ng} / \mathrm{kg} /$ min every six hours). The first two titrations of inhaled treprostinil (three and six breaths) would be expected to provide concentrations less than a parenteral dose of $20 \mathrm{ng} /$ $\mathrm{kg} / \mathrm{min}$; however, both patients were already receiving SC treprostinil as the doses were being down-titrated. Ultimately, both transitions occurred safely over an accelerated period of time (three to four days). Although in-transition hemodynamic parameters for the first patient were not available, in-transition hemodynamic data for Patient 2 suggested that the transition was successful because no deterioration in hemodynamic parameters was evident. Interestingly, longer-term post-inhaled treprostinil hemodynamic data at five months continued to show benefits of inhaled therapy. These hemodynamic findings were validated with observations of 6-MWD, functional class and NT-proBNP concentration.

In terms of identifying patients who may be appropriate candidates for transition to inhaled from parenteral treprostinil, we emphasize that this approach should not be employed routinely in stable patients who are tolerating parenteral prostacyclin therapy given the proven benefits of IV and SC treprostinil. In addition, we risk stratified the patients transitioned to inhaled treprostinil using the multivariable risk prediction algorithm derived from the REVEAL PAH registry. ${ }^{[18]}$ Based on this multivariable risk scoring system, both patients would have a predicted oneyear survival $>95 \%$ prior to the transition and we noted that REVEAL scores did not change appreciably at follow-up after the transition.

We recommend that offering transition to inhaled treprostinil should be restricted to carefully selected patients who have preserved functional capacity (WHO Functional Class II), stable clinical course prior to the transition with low risk of one-year mortality based on contemporary risk prediction algorithms such as REVEAL and ideally who are on relatively lower doses of treprostinil $(<40 \mathrm{ng} / \mathrm{kg} / \mathrm{min})$.

We base the latter recommendation on the PKs of inhaled treprostinil and the effective Cmax achieved with the maximal dose of inhaled therapy as well as prior clinical studies in transitioning from IV prostacyclin to oral bosentan, in which the likelihood of successful transition was higher than in those on lower doses of prostacylcin. ${ }^{[19]}$

Patients being considered for transition should also have experienced major complications of parenteral prostacyclin therapy such as sepsis, recurrent abscesses, intractable infusion site pain, or inability to appropriately manage the infusion system. Transition, if contemplated, should be made in the hospital setting at an experienced PAH center and preferably with continuous hemodynamic monitoring if the patient is on higher doses of parenteral treprostinil. In addition, close clinical follow-up as an outpatient with biomarker assessment, 6-MWT and WHO functional class is mandatory to ensure stability post-transition.

Because of limitations in how high the dose of inhaled treprostinil can be escalated (there are no data for doses $>54 \mu$ g q.i.d.), particular caution is recommended for physicians with patients who are transitioning from high doses of parenteral treprostinil. For this patient group, unless there is an emergent contraindication to parenteral therapy, the best course of action may be to continue parenteral therapy. These case evaluations are limited by the lack of PK data from our patients prior to their transition to inhaled treprostinil. Indeed, the patient receiving the higher dose (61 ng/kg/min) may have had lower plasma concentrations than expected because of altered site integrity and related absorption due to SC abscesses.

In summary, transitioning from SC to inhaled treprostinil can be achieved successfully in carefully selected patients with preservation of functional capacity, hemodynamics and quality of life. The transition protocol employed was relatively simple and was based on established PK characteristics of treprostinil administered by continuous infusion and by intermittent inhalation. In this clinical setting of drug transition (parenteral to inhaled), we defined success as "maintenance of disease status without clinical deterioration." Based on this definition, the cases presented here were considered to be successful. These cases provide guidance to physicians who are experienced in managing patients with PAH on prostanoid therapy and are considering transitioning selected patients from parenteral therapy to inhaled therapy.

\section{REFERENCES}

1. Farber HW, Loscalzo J. Pulmonary arterial hypertension. N Engl J Med 2004;351:1655-65.

2. McLaughlin VV, Archer SL, Badesch DB, Barst RJ, Farber HW, Lindner JR, et al. ACCF/AHA 2009 expert consensus document on pulmonary hypertension: A report of the American College of Cardiology Foundation Task Force on Expert Consensus Documents and the American Heart Association: Developed in collaboration with the American College of Chest Physicians, American Thoracic Society, Inc. and the Pulmonary Hypertension Association. Circulation 2009;119:2250-94.

3. Barst RJ, Galie N, Naeije R, Simonneau G, Jeffs R, Arneson C, et al. Long-term outcome in pulmonary arterial hypertension patients treated with subcutaneous treprostinil. Eur Respir J 2006;28:1195-203.

4. Hiremath J, Thanikachalam S, Parikh K, Shanmugasundaram S, Bangera S, Shapiro L, et al. Exercise improvement and plasma biomarker changes with intravenous treprostinil therapy for pulmonary arterial hypertension: A placebo-controlled trial. J Heart Lung Transplant 2010; 29:137-49.

5. Lang I, Gomez-Sanchez M, Kneussl M, Naeije R, Escribano P, Skoro-Sajer N, et al. Efficacy of long-term subcutaneous treprostinil sodium therapy in pulmonary hypertension. Chest 2006;129:1636-43.

6. Tapson VF, Gomberg-Maitland M, McLaughlin VV, Benza RL, Widlitz AC, 
Krichman A, et al. Safety and efficacy of IV treprostinil for pulmonary arterial hypertension: A prospective, multicenter, open-label, 12-week trial. Chest 2006;129:683-8.

7. Simonneau G, Barst RJ, Galie N, Naeije R, Rich S, Bourge RC, et al. Continuous subcutaneous infusion of treprostinil, a prostacyclin analogue, in patients with pulmonary arterial hypertension: A double-blind, randomized, placebo-controlled trial. Am J Respir Crit Care Med 2002;165:800-4.

8. Kallen AJ, Lederman E, Balaji A, Trevino I, Petersen EE, Shoulson R, et al. Bloodstream infections in patients given treatment with intravenous prostanoids. Infect Control Hosp Epidemiol 2008;29:342-9.

9. McLaughlin VV, Benza RL, Rubin LJ, Channick RN, Voswinckel R, Tapson VF, et al. Addition of inhaled treprostinil to oral therapy for pulmonary arterial hypertension: A randomized controlled clinical trial. J Am Coll Cardiol 2010;55:1915-22.

10. Laliberte K, Arneson C, Jeffs R, Hunt T, Wade M. Pharmacokinetics and steady-state bioequivalence of treprostinil sodium (Remodulin) administered by the intravenous and subcutaneous route to normal volunteers. J Cardiovasc Pharmacol 2004;44:209-14.

11. Barst RJ, Rubin LJ, Long WA, McGoon MD, Rich S, Badesch DB, et al. A comparison of continuous intravenous epoprostenol (prostacyclin) with conventional therapy for primary pulmonary hypertension. N Engl J Med 1996;334:296-301.

12. de Jesus Perez VA, Rosenzweig E, Rubin LJ, Poch D, Bajwa A, Park M, et al. Safety and efficacy of transition from systemic prostanoids to inhaled treprostinil in pulmonary arterial hypertension. Am J Cardiol 2012;110:1546-50.

13. Nelsen AC, Laliberte KJ, Zaccardelli DS, Gotzkowsky SK. Pharmacokinetics of inhaled treprostinil sodium in healthy volunteers. Am J Respir Crit Care Med 2010;181:A3348.

14. Voswinckel R, Enke B, Reichenberger F, Kohstall M, Kreckel A, Krick S, et al. Favorable effects of inhaled treprostinil in severe pulmonary hypertension: Results from randomized controlled pilot studies. J Am Coll Cardiol 2006;48:1672-81.

15. Wade M, Baker FJ, Roscigno R, DellaMaestra W, Hunt TL, Lai AA. Absolute bioavailability and pharmacokinetics of treprostinil sodium administered by acute subcutaneous infusion. J Clin Pharmacol 2004;44:83-8.

16. Wade M, Baker FJ, Roscigno R, DellaMaestra W, Hunt TL, Lai AA. Pharmacokinetics of treprostinil sodium administered by 28-day chronic continuous subcutaneous infusion. J Clin Pharmacol 2004; 44:503-9.

17. McSwain CS, Benza R, Shapiro S, Hill N, Schilz R, Elliott CG, et al. Dose proportionality of treprostinil sodium administered by continuous subcutaneous and intravenous infusion. J Clin Pharmacol 2008;48:19-25.

18. Benza RL, Gomberg-Maitland M, Miller DP, Frost A, Frantz RP, Foreman AJ, et al. The REVEAL Registry riskscore calculator in patients newly diagnosed with pulmonary arterial hypertension. Chest 2012;141:354-62.

19. Steiner MK, Preston IR, Klinger JR, Criner GJ, Waxman AB, Farber HW, et al. Conversion to bosentan from prostacyclin infusion therapy in pulmonary arterial hypertension: A pilot study. Chest 2006;130:1471-80.

Source of Support: Raymond L. Benza received research funding from the sponsor (United Therapeutics Corporation), Lung Rx, Pfizer, Gilead Sciences and Actelion. He is a paid advisor for the sponsor and serves on advisory boards for the sponsor and Actelion. He is a paid speaker for the sponsor, Gilead Sciences and Actelion. He does not have equity interest in any pharmaceutical company. Srinivas Murali has received research funding from the sponsor (United Therapeutics Corporation), Lung Rx, Pfizer, Gilead Sciences and Actelion. He is a paid speaker for the sponsor, Gilead Sciences and Actelion. He does not have equity interest in any pharmaceutical company. George Sokos is a paid speaker for the sponsor, Gilead Sciences and Actelion. He does not have equity interest in any pharmaceutical company. The site's principal investigators designed and wrote the manuscript without compensation. Assistance with editing was provided under the direction of the authors by Larry Nelson and Chris Lawrence, MedThink Communications, with support from United Therapeutics Corporation. United Therapeutics Corporation did not fund the study nor participate in data collection, analysis, or interpretation. The company waived the right to approve or disapprove publication of the completed manuscript. Conflict of Interest: None declared. 